\title{
Nonlinear model of short-scale electrodynamics in the auroral ionosphere
}

\author{
J.-M. A. Noël ${ }^{1}$, J.-P. St.-Maurice ${ }^{1}$, P.-L. Blelly ${ }^{2}$ \\ ${ }^{1}$ Department of Physics and Astronomy, The University of Western-Ontario, London, Ontario, Canada \\ ${ }^{2}$ Centre d'étude spatiale des rayonnements, Toulouse, France
}

Received: 24 January 2000 / Revised: 10 May 2000 / Accepted: 5 June 2000

\begin{abstract}
The optical detection of auroral subarcs a few tens of $m$ wide as well as the direct observation of shears several $\mathrm{m} / \mathrm{s}$ per $\mathrm{m}$ over $\mathrm{km}$ to sub $\mathrm{km}$ scales by rocket instrumentation both indicate that violent and highly localized electrodynamics can occur at times in the auroral ionosphere over scales $100 \mathrm{~m}$ or less in width. These observations as well as the detection of unstable ion-acoustic waves observed by incoherent radars along the geomagnetic field lines has motivated us to develop a detailed time-dependent two-dimensional model of short-scale auroral electrodynamics that uses current continuity, Ohm's law, and 8-moment transport equations for the ions and electrons in the presence of large ambient electric fields to describe wide auroral arcs with sharp edges in response to sharp cut-offs in precipitation (even though it may be possible to describe thin arcs and ultra-thin arcs with our model, we have left such a study for future work). We present the essential elements of this new model and illustrate the model's usefulness with a sample run for which the ambient electric field is $100 \mathrm{mV} / \mathrm{m}$ away from the arc and for which electron precipitation cuts off over a region $100 \mathrm{~m}$ wide. The sample run demonstrates that parallel current densities of the order of several hundred $\mu \mathrm{A} \mathrm{m}^{-2}$ can be triggered in these circumstances, together with shears several $\mathrm{m} / \mathrm{s}$ per $\mathrm{m}$ in magnitude and parallel electric fields of the order of $0.1 \mathrm{mV} / \mathrm{m}$ around $130 \mathrm{~km}$ altitude. It also illustrates that the local ionospheric properties like densities, temperature and composition can strongly be affected by the violent localized electrodynamics and vice-versa.
\end{abstract}

Key words: Ionosphere (auroral ionosphere, electric fields and currents, ionosphere-magnetosphere interactions)

Correspondence to: J.-P. St.-Maurice

e-mail: jstmauri@julian.uwo.ca

\section{Introduction}

The existence of extremely narrow auroral structures has been coming to light over the years. By extremely narrow we mean structures that have widths of a few meters (ultra-thin arcs) up to a few tens of meters (thin arcs). Their existence has been inferred using rockets, (e.g., Marklund et al., 1982; Earle et al., 1989), satellites, (e.g., Cerisier et al., 1987; Berthelier et al., 1988) and by optical means, (e.g., Maggs and Davis, 1968; Trondsen and Cogger, 1997).

Using a so-called "Portable Auroral Imager", Trondsen (1998) has shown from images that auroral structures can at times be extremely narrow. To just quote from Trondsen's web page: "The Portable Auroral Imager (PAI) provides high temporal resolution optical measurements of auroral phenomena. High sensitivity is achieved through the use of modern image intensifier technology in conjunction with a charge-coupled device. The imager is equipped with a standard C-mount lens adapter, allowing the use of a wide range of optical assemblies from telescopes to all-sky lenses. Field of view/spatial resolution can thus be tailored closely to match that needed best to study the phenomenon at hand and to provide supporting measurements for any other instrument simultaneously employed. Data is stored for later digitization using high-resolution analog video recording equipment". Using this Portable Auroral Imager Trondsen (1998) has shown that auroral substructures can be smaller than a few tens of $m$ wide. These results came from an imager fielded at Rabbit Lake in northern Saskatchewan, Canada. This site was chosen for its location within the auroral zone and for the additional instrumental support coming from a permanently installed all-sky camera and magnetometer as well as other scientific instrumentation. During campaigns in 1994 and 1995 Trondsen observed twelve examples of extremely narrow arcs 20 to $100 \mathrm{~m}$ wide that were stationary enough for their extreme thinness to be properly resolved. He concluded that even though 
more data was obviously needed for meaningful statistics the existing data set suggested that subarc thicknesses were usually found ranging from 50 to $70 \mathrm{~m}$ in width.

Trondsen's results confirm and add to earlier results obtained from Maggs and Davis (1968), who used an orthicon television system to obtain the thicknesses of 581 auroral structures over College, Alaska. Maggs and Davis found, assuming a lower border height of $100 \mathrm{~km}$ for the structures, that the thickness ranged from the instrumental limit of $70 \mathrm{~m}$ up to $4440 \mathrm{~m}$. A striking point was that fifty percent of the 581 measured structures had a thickness less than $230 \mathrm{~m}$. Maggs and Davis also noted that the auroral structures had a tendency to become thinner as their brightness increased.

Armed with these examples of optical observations, one cannot ignore the fact that thin auroral structures exist. It seems logical to infer from this that intense latitudinal gradients must also be present in the plasma density. As a result, we surmise that sharp gradients in the conductivity with scale sizes at least comparable to the thickness of the narrow optical structures themselves can be postulated to exist even in wide arcs. As we now discuss, this hypothesis seems to be supported by certain rocket and satellite data.

Turning now to some in-situ observations, we note that Marklund et al. (1982) studied a discrete auroral arc with detectors on the S23L1 rocket in coordination with ground-based optical measurements. In their study they observed a dramatic decrease in the northward component of the electric field as the rocket entered the arc. On the other hand, the northward electric field fell from $115 \mathrm{mV} / \mathrm{m}$ outside the arc to approximately $20 \mathrm{mV} / \mathrm{m}$ inside the arc. By contrast, the eastward component of the electric field remained nearly constant between 5 and $20 \mathrm{mV} / \mathrm{m}$. In addition, Marklund et al. observed that the electron density increased while the electric field strength was decreasing. The anti-correlation between the electric field and the electron density across the arc indicated a southward polarization electric field within the arc. Using the following assumptions: (1) there were no conductivity gradients along the arc; (2) $\nabla \times E=0$, i.e., no dramatic changes in the magnetic field were measured on the ground; (3) either $E_{x}$ or $E_{y}$ component of the electric field was constant when moving along the arc, they concluded that there had to be a pair of very intense current sheets carrying current densities of the order of $500 \mu \mathrm{A} / \mathrm{m}^{2}$ when the rocket was crossing the arc. This current density is very large when compared to the accepted average value of $1-10 \mu \mathrm{A} / \mathrm{m}^{2}$.

Another signature which has been shown to be related directly to intense parallel current structures is the presence of velocity shears reaching several meters per second per meter in the plasma drift. An example from Earle et al. (1989) showed an $F$ region auroral structure where the electric field abruptly changed by $180 \mathrm{mV} / \mathrm{m}$ within a distance of 363 meters, yielding a velocity shear of $11 \mathrm{~m} / \mathrm{sec}$ per meter. Using a typical value of the Pedersen conductivity of the E-region in the range $1 \times 10^{-5}-1 \times 10^{-4} \mathrm{mho} / \mathrm{m}$, a neutral scale height of $10 \mathrm{~km}$ and the velocity shear measured by Earle et al. (1989), a "back of envelope" calculation using, $J_{\|} / H_{n}=\sigma_{\mathrm{Ped}} \Delta E_{x} / \Delta x$ yields an estimate for the current density that could be as high as $500 \mu \mathrm{A} / \mathrm{m}^{2}$. Earle et al. (1989) even observed shears of $25 \mathrm{~m} / \mathrm{sec}$ per meter implying that the estimated parallel current density could actually even be twice as large at times.

Satellites have also hinted to the existence of intense field-aligned current densities. For instance, Cerisier et al. (1987) in trying to interpret a magnetic pulse measured by a magnetometer aboard the AUREOL 3 low altitude satellite concluded that they could have been observing current densities as high as $500 \mu \mathrm{A} / \mathrm{m}^{2}$ in a current sheet $20 \mathrm{~m}$ wide. A similar conclusion was reached by Berthelier et al. (1988) in their study of similar data sets.

Radar observations have also suggested the existence of extremely intense, localized current densities. The clearest indication has come from anomalies in incoherent scatter radar measurements. For instance, Foster et al. (1991), Rietveld et al. (1991) and Collis et al. (1991) observed radar echoes which had substantial enhancements in one or both of the ion-acoustic shoulders. This was interpreted as meaning that the ion-acoustic waves were either unstable or very close to being unstable. At the radar frequency of interest, threshold calculations for a two-stream instability for the conditions under consideration produced fieldaligned current densities carried by thermal electrons which had to be in excess of $1000 \mu \mathrm{A} / \mathrm{m}^{2}$ at times. This inference stimulated the search for alternative mechanisms (e.g., Forme, 1993; Whalund et al., 1992) as well as for seriously considering the possibility that ionospheric auroral electrodynamics could be far more violent at times than had previously been thought (St.-Maurice et al., 1996).

The observations of Rietveld et al. (1991) and Collis et al. (1991) were the motivation behind a first study of local electrodynamics by our group (St.-Maurice et al., 1996). Using a simple model, we calculated the effects of horizontal variations in the precipitation pattern as well as of variations in the horizontal conductivities on the in-situ generation of intense field-aligned currents. The model was two-dimensional and time-independent. Using current continuity requirements, a thermal ionospheric current system was calculated in response to a nonthermal current source (i.e., energetic particle precipitation). The current system and associated potential were obtained for a Cartesian geometry across an elongated arc. An important limitation was its timeindependence: the model ionosphere was not allowed to react to the presence of new electric fields and associated heating effects (as we show below the time-dependent ionospheric response actually provides a positive feedback by which intense parallel currents actually intensify).

St.-Maurice et al. (1996) focused on two types of current sources. The first source of thermal electron currents was simply the parallel currents carried by energetic electrons. The parallel current density pattern 
was modeled using a Lorentzian model of the latitudinal dependence of the precipitating region. Thermal electrons were responding to the parallel electric fields produced when the energetic electrons were stopped by the neutral atmosphere. If the nonthermal particles were stopped at too high an altitude, the thermal electrons were sent back up toward the magnetic field line to a considerable extent, creating a net current (downgoing nonthermal electrons minus upgoing thermal electrons) that was small by comparison to the current carried by either type. At the other extreme, if the nonthermal electrons were so energetic that they would make it to the conducting $\mathrm{E}$ region before being stopped, most of the current closure was done horizontally, and the parallel currents carried by thermal electrons were minimal. For intermediate situations, thermal electrons were flowing away from the region of energetic electron deposition, that is, upward above the stopping altitude and downward below that altitude.

The second type of current source considered by St.-Maurice et al. (1996) was linked to the divergence in the current density produced by horizontal conductivity gradients in the presence of a large-scale ambient convection electric field. With this second model, St.Maurice et al. (1996) were able to have thermal electrons carry field-aligned current densities ranging from $213 \mu \mathrm{A} / \mathrm{m}^{2}$ up to $750 \mu \mathrm{A} / \mathrm{m}^{2}$. They got by the same token velocity shears of up to $6 \mathrm{~m} / \mathrm{sec}$ per meter. It was concluded that in order to generate parallel fields and thermal electron motion intense enough to destabilize ion-acoustic waves along the geomagnetic field line, $100 \mathrm{~m}$ gradient scale lengths or smaller were needed.

The present work is a first extension of the work begun by St.-Maurice et al. (1996) and will still be used initially for simplicity to describe the electrodynamics of stationary wide arcs with sharp boundaries (even though we show in the Appendix that there is no fundamental difficulty in modeling even fast moving ultra-thin arcs with our approach). Our new model uses a sophisticated set of nonlinear time-dependent transport equations that couples with an electrostatic code that solves for the null current divergence condition. Central to our paper is the introduction of an alternative technique to solve the current continuity equation. While we still use a steady state current continuity equation because of the very short time scales involved in establishing perturbed electric fields in response to conductivity changes (e.g., St.-Maurice et al., 1996) we have had to revamp the numerical method so as to solve that equation more efficiently. The new method, which we describe as "pseudo-spectral" is based on a Fourier analysis of the variables in the plane perpendicular to the geomagnetic field. It converges relatively quickly. This allows us to iterate back and forth between the electrodynamics and the ionospheric dynamics, and indeed to proceed with time-dependent studies. With the new model we are therefore able to study the changes in the composition and energetics of the ionosphere associated with the electrodynamics and indeed the feedback between the two. To that goal we now also use a much more comprehensive transport model of the auroral iono- sphere, based on the numerical model developed at CESR and elsewhere over the years.

The present paper focuses primarily on the model itself. We give details of the physical processes that have been included in the calculation and the numerical methods that were employed. Note that the bulk of the presentation deals with the new electrodynamical coding since the transport part of the model has been described and used repeatedly elsewhere. Only in the latter portion of the paper do we present some initial results from a couple of model runs.

We proceed with the rest of our presentation as follows: in Sect. 2, we introduce our basic electrostatic equations and describe our conductivity model. In the process we also discuss some examples of possible feedback that could occur during our simulations. Sect. 3 is devoted to a description of the numerical technique we used to compute the electrostatic potential as well as to a description of the boundary conditions that were applied. In Sect. 4 we describe the transport model and the relevant equations that were modified to complete the coupling with the electrodynamical model. Sect. 5 contains a description of the latitudinal pattern that we used for the energetic electron precipitation as well as a description of how the models were coupled. We present initial results from a couple of model runs in Sect. 6. We end with a summary and conclusion in Sect. 7.

\section{The electrodynamics model - basic equations}

\subsection{The electrostatic equation}

As was discussed in the introduction, we wish to investigate the possibility of positive feedback between electrodynamics and the composition and energetics of the ionosphere in the presence of sharp horizontal transitions in the conductivities. For the present work we will limit ourselves to the simple case of wide stationary arcs with sharp (100 to $200 \mathrm{~m}$ ) precipitation cut-offs. We will not explore in the present study other possibilities like thin arcs and/or moving arcs even though there is no fundamental difficulty with our present code to model these more dynamical objects, as long as they are elongated (see the Appendix).

The electrodynamical part of our model is based on the approach suggested by St.-Maurice et al. (1996). It starts with the assumption of a divergence-free current density. This can be interpreted as either pre-supposing that the time scales exceed the electron collision time or that we are averaging out the effect of plasma oscillations if the geophysical time scales are shorter than the electron collision time (see discussion in St.-Maurice et al., 1996). As a result we can write

$\nabla \cdot \mathbf{J}=0$

Recall again that Eq. 1 does not mean that the model is time-independent. Rather, the lack of a time dependence at the electrodynamics level simply means that the response scales are so fast that the perturbed electric 
fields and resulting currents are adjusting essentially instantaneously to any temporal change in conductivity or precipitation input.

In a deviation from what others might normally do at this point, we divide the current density, $\mathbf{J}$, into three parts: a source term from precipitation, $\mathbf{J}_{s}^{p}$, a second source term caused by the presence of a quasi-uniform background electric field in a region of strong horizontal gradients, and a "thermal response term", $\mathbf{J}_{\text {th }}$. The source term from precipitation simply describes the currents carried by precipitating fluxes of energetic particles. The behavior of that current source is modeled by calculating the vertical variations in the precipitating flux according to a first principles model that includes all forms of interactions with the neutrals and other charged particles (Lilensten et al., 1989; Lummerzheim and Lilensten, 1994). Any particular choice of energetic electron flux can be specified at a sufficiently large distance from the ionosphere.

We describe the rest of the current with a simple Ohm's law that is,

$\mathbf{J}=\boldsymbol{\sigma} \cdot \mathbf{E}$

where $\boldsymbol{\sigma}$ is the classical conductivity tensor, and $\mathbf{E}$ is the electric field. As usual, the conductivity tensor is given by

$\boldsymbol{\sigma}=\left(\begin{array}{ccc}\sigma_{P} & \sigma_{H} & 0 \\ -\sigma_{H} & \sigma_{P} & 0 \\ 0 & 0 & \sigma_{\|}\end{array}\right)$

where $\sigma_{P}, \sigma_{H}$ and $\sigma_{\|}$are the Pedersen, Hall and parallel conductivities respectively.

We assume that a quasi-uniform background electric field already exists before our arc is introduced in the system. We label this part of the electric field as $\mathbf{E}_{0}$. That field is taken to be in the north-south direction, perpendicular to the elongated arc. A disturbance electric field is introduced next as an arc is inserted into the system. This is done by having the arc produce a local change in conductivity which in turn drives new currents and a disturbance electric field. We therefore divide the electric field into two parts, namely, $\mathbf{E}=\mathbf{E}_{0}-\nabla \phi$. In this expression $\mathbf{E}_{0}$ is a constant and $\phi$ is the electrostatic potential of the disturbance field. As a result of these considerations we write

$\nabla \cdot \mathbf{J}=\nabla \cdot\left(\boldsymbol{\sigma} \cdot \mathbf{E}_{0}\right)-\nabla \cdot(\boldsymbol{\sigma} \cdot \nabla \phi)+\nabla \cdot \mathbf{J}_{s}^{p}$

where we recall that $\mathbf{J}_{s}^{p}$ is the current carried by the precipitating electrons. Since $\mathbf{E}_{0}$ is uniform while $\boldsymbol{\sigma}$ is given by a separate transport model, we can also view the first term on the right-hand side of Eq. 4 as another source term. The ambient electric field being perpendicular to the geomagnetic field in that case we can then write

$\nabla \cdot\left(\boldsymbol{\sigma} \cdot \mathbf{E}_{0}\right)=E_{x}^{0} \frac{\partial}{\partial x} \sigma_{P}(x, z)+E_{y}^{0} \frac{\partial}{\partial x} \sigma_{H}(x, z)$

where the terms $\partial \sigma_{P, H}(x, z) / \partial x$ are the horizontal northsouth gradients in the Pedersen/Hall conductivities introduced by the edge of the precipitating region. The second term on the right-hand side of Eq. 4 is what we define as the divergence of "thermal response" current term, $\mathbf{J}_{\text {th }}$. Note that Eqs. 1, 4 and 5 are valid even when the arc moves, as long as the motion is given by $E_{y}^{0} / B$ in the $x$ direction (see Appendix for more).

As a result of the above definitions and Eq. 1 we end up having to solve the following equation for the potential $\phi$ :

$$
\nabla \cdot(\boldsymbol{\sigma} \cdot \nabla \phi)=E_{x}^{0} \frac{\partial}{\partial x} \sigma_{P}(x, z)+E_{y}^{0} \frac{\partial}{\partial x} \sigma_{H}(x, z)+\nabla \cdot \mathbf{J}_{s}^{p}
$$

In general the source term due to $\mathbf{J}_{s}^{p}$ is far too weak to produce the hundreds of $\mu \mathrm{A} \mathrm{m}^{-2}$ that we wish to study in the present work. Therefore, we drop that term from the rest of this paper. Likewise, in the context of the fact that the motion of arcs is often far smaller than the ambient $\mathbf{E}_{0} \times \mathbf{B}$ drift, we will drop $E_{y}^{0}$ for our initial study. Notice that our dropping of the $\mathbf{J}_{s}^{p}$ term does not mean that the parallel currents carried by energetic electrons are not important in our work. However, they manifest themselves through the other current source terms containing $E_{x}^{0}$ and $E_{y}^{0}$. These terms are strongly linked to precipitation through the intense horizontal conductivity gradient scales that we will consider here.

To be more specific still about the model, we now write our last equation completely in terms of our coordinates. We use a Cartesian coordinate system where $z$ is aligned with the Earth's magnetic field (vertical) and $x$ is along the horizontal direction. We also assume that the precipitation pattern, or arc, is elongated in the $y$ direction and has negligible motion, which means that we set $\partial / \partial y=0$ and $E_{y}^{0}=0$. After dropping the $\mathbf{J}_{s}^{p}$ term as well, we then end up with

$$
\begin{aligned}
& \frac{\partial}{\partial x}\left(\sigma_{P}(x, z) \frac{\partial \phi(x, z)}{\partial x}\right)+\frac{\partial}{\partial z}\left(\sigma_{\|}(x, z) \frac{\partial \phi(x, z)}{\partial z}\right) \\
& \quad \approx E_{x}^{0} \frac{\partial \sigma_{P}(x, z)}{\partial x}
\end{aligned}
$$

where $\phi(x, z)$ is the electric potential that we are solving for. The horizontal dependency arises from the precipitation pattern that we impose initially and the associated changes in the conductivities with position.

\subsection{Modeling of the conductivities}

In Eq. 7 the conductivities are written as functions of latitudinal position, $x$, as well as altitude, $z$. The standard expressions for the conductivities as a function of altitude may be found in a number of references (e.g., Hargreaves, 1992; Kelley, 1989; Kivelson and Russel, 1995). They read,

$\sigma_{\|}(z)=e^{2}\left[\frac{n_{e}(z)}{m_{e} v_{e}(z)}+\sum_{i}^{\text {ions }} \frac{n_{i}(z)}{m_{i} v_{i}(z)}\right]$

and, 


$$
\begin{aligned}
\sigma_{P}(z)= & e^{2}\left[\frac{n_{e}(z)}{m_{e} v_{e}(z)} \frac{v_{e}^{2}(z)}{v_{e}^{2}(z)+\Omega_{e}^{2}(z)}\right. \\
& \left.+\sum_{i}^{\text {ions }} \frac{n_{i}(z)}{m_{i} v_{i}(z)} \frac{v_{i}^{2}(z)}{v_{i}^{2}(z)+\Omega_{i}^{2}(z)}\right]
\end{aligned}
$$

From these expressions we can see that if there exists any structuring in the temperature and/or the plasma density, a structuring in the conductivities will result. In particular, any modification in the ionospheric properties due to the effects of $\mathbf{J}_{\text {th }}$ will create perturbations in the conductivities in the vicinity of the precipitation.

The spatial variations in the conductivities have been written in the form

$\sigma_{P}(x, z)=\sigma_{P}^{\infty}(z)[1+h(x, z)]$

and,

$\sigma_{\|}(x, z)=\sigma_{\|}^{\infty}(z)[1+g(x, z)]$

where $\sigma_{\|}^{\infty}(z)$ and $\sigma_{P}^{\infty}(z)$ are constant background values of the parallel and Pedersen conductivities. The perturbations in the conductivities of the precipitating regions are modeled by $h(x, z)$ and $g(x, z)$. The boundary conditions are such that, far away from the precipitation region the functions $h(x, z)$ and $g(x, z)$ become negligible. This means that both $\sigma_{\|}^{\infty}(z)$ and $\sigma_{P}^{\infty}(z)$ can be determined from Eqs. 8 and 9 respectively, using the background (unperturbed) plasma conditions.

The conductivity perturbations themselves $[h(x, z)$ and $g(x, z)$ in Eqs. 10 and 11] can be computed from the density enhancements and electron temperature changes induced by the precipitation and the ensuing changes in the thermal electron currents and in the electric field. In that sense in fact the functions $g$ and $h$ are timedependent since the densities change fairly rapidly after the onset of precipitation at time $t=0$. The horizontal gradient in the Pedersen conductivity is then used as the source term in Eq. 7. From its knowledge, the timedependent electric potential (which, unlike the source term, depends on $\sigma_{\|}$as well) can then be computed. This information is fed back to the transport equations and the iteration continues in that way as time advances.

\subsection{Examples of positive feedback mechanism}

As we just illustrated for the functions $g$ and $h$, the reason for using a coupled model is that one process can affect the others. This makes the system nonlinear in the sense that, while we march in time, the currents are changing the medium, which then modifies the structure of the currents and so on. The effect of this coupling was not considered in the steady state study by St.-Maurice et al. (1996). As a specific example consider the effects of chemistry on the currents in our present model: the creation of intense parallel currents due to sharp horizontal cut-offs in the plasma density can heat the electrons substantially. The elevated electron temperatures in turn affect the chemical composition of the ionosphere. The chemical recombination of $\mathrm{NO}^{+}$, which reads
$\mathrm{NO}^{+}+e \longrightarrow \mathrm{O}+\mathrm{N}+ \begin{cases}0.38 \mathrm{eV}, & \Gamma=0.78 \\ 2.75 \mathrm{eV}, & \Gamma=0.22\end{cases}$

has a chemical reaction rate given by (Rees, 1989):

$\alpha=4.2 \times 10^{-7}\left(\frac{T_{e}}{300}\right)^{-0.85} \times \Gamma$

Thus, as the electron temperature rises, the recombination rate decreases. This means an enhancement in the plasma density in the region of high parallel current density. The localized plasma density enhancement in turn creates a larger horizontal gradient in the Pedersen conductivity. As can be inferred from Eq. 7, this further increases the parallel currents, which heat the electrons further. The hotter electrons then lower the recombination rate and the whole process can repeat itself in a positive feedback loop.

A second way the plasma density and therefore the conductivity can be affected is through the creation of perpendicular electric field perturbations. Perpendicular electric fields heat the ions through friction with the neutrals. That means that in regions where $E_{\perp}$ is large there is an increase in ion heating. The resulting ion temperature in turn affects the composition. For example, take the reaction,

$\mathrm{O}^{+}+\mathrm{N}_{2} \longrightarrow \mathrm{NO}^{+}+\mathrm{N}+1.10 \mathrm{eV}$

Its chemical reaction rate depends very sensitively on the effective temperature, $T_{\text {eff }}$ (e.g., St.-Maurice and Laneville, 1998). This effective temperature is given by

$T_{\text {eff }}=\frac{m_{n}}{m_{i}+m_{n}}\left(\frac{m_{i} U^{2}}{3 k_{b}}+T_{i}-T_{n}\right)+T_{n}$

where $m_{n}$ and $m_{i}$ are the masses of the neutral and ion reactants respectively, $k_{b}$ is the Boltzmann constant, $T_{i}$ and $T_{n}$ are the ion and neutral temperatures respectively, and $U$ is the magnitude of the relative drift between the ions and neutral reactants.

In the expression for the effective temperature we note that as $T_{i}$ increases through frictional heating, the effective temperature also increases. This means that the ionosphere increases the "cooking" rate of $\mathrm{O}^{+}$into $\mathrm{NO}^{+}$. With the increase in the $\mathrm{NO}^{+}$production rate, the reaction in Eq. 12 is enhanced further. The net effect is a conversion to molecular ions and the reduction in the electron density in regions of large electric fields.

From these two examples it should be clear that the coupling between electrodynamics and the dynamics of the ionosphere can be important.

\section{Solving the electrostatic equation}

\subsection{The pseudo-spectral method}

We now describe the "pseudo-spectral" technique used to solve Eq. 7. The basic idea is to use a Fourier transform of Eq. 7 along $x$ while solving for the altitude dependence with a finite difference technique. The pseudo-spectral method was chosen primarily because it is computationally faster. For instance, the full finite 
difference technique used by St.-Maurice et al. (1996) required millions of iterations before convergence was obtained. In that study, a long time was therefore required to do a single simulation, making a comprehensive study of the kind undertaken here impractical.

At the heart of the method we take a Fourier transform of Eq. 7 with respect to $x$. However, because of the presence of product terms the method becomes nonlinear in a Fourier sense. This introduces complications that must now be discussed. To start with, once the transform is done we obtain the following expression for the first term on the left-hand side of Eq. 7:

$$
\begin{aligned}
& \int_{-\infty}^{\infty}\left[\frac{\partial}{\partial x}\left(\sigma_{P}(x, z) \frac{\partial \phi(x, z)}{\partial x}\right)\right] \mathrm{e}^{-i k x} \mathrm{~d} x \\
& =-\frac{1}{2 \pi} \int_{-\infty}^{\infty} k k^{\prime \prime \prime} \sigma_{P}\left(k-k^{\prime \prime \prime}, z\right) \phi\left(k^{\prime \prime \prime}, z\right) \mathrm{d} k^{\prime \prime \prime}
\end{aligned}
$$

The second term on the left-hand side of Eq. 7 may likewise be written as,

$$
\begin{aligned}
\int_{-\infty}^{\infty} & {\left[\frac{\partial}{\partial z}\left(\sigma_{\|}(x, z) \frac{\partial \phi(x, z)}{\partial z}\right)\right] \mathrm{e}^{-i k x} \mathrm{~d} x } \\
= & \frac{1}{2 \pi} \int_{-\infty}^{\infty} \sigma_{\|}\left(k-k^{\prime \prime \prime}, z\right) \frac{\partial^{2} \phi\left(k^{\prime \prime \prime}, z\right)}{\partial z^{2}} \mathrm{~d} k^{\prime \prime \prime} \\
& +\frac{1}{2 \pi} \int_{-\infty}^{\infty} \frac{\partial \sigma_{\|}\left(k-k^{\prime \prime \prime}, z\right)}{\partial z} \frac{\partial \phi\left(k^{\prime \prime \prime}, z\right)}{\partial z} \mathrm{~d} k^{\prime \prime \prime}
\end{aligned}
$$

while the right-hand side may be written as,

$$
\int_{-\infty}^{\infty} E_{x}^{0} \frac{\partial \sigma_{P}(x, z)}{\partial x} \mathrm{e}^{-i k x} \mathrm{~d} x=i k \sigma_{P}(k, z) E_{x}^{0}
$$

The conductivities in the last three equations can be rewritten by taking the Fourier transforms of Eqs. 10 and 11 to get

$\sigma_{\|}\left(k-k^{\prime \prime \prime}, z\right)=\sigma_{\|}^{\infty}(z)\left[2 \pi \delta\left(k^{\prime \prime \prime}-k\right)+G\left(k-k^{\prime \prime \prime}, z\right)\right]$

and

$\sigma_{P}\left(k-k^{\prime \prime \prime}, z\right)=\sigma_{P}^{\infty}(z)\left[2 \pi \delta\left(k^{\prime \prime \prime}-k\right)+H\left(k-k^{\prime \prime \prime}, z\right)\right]$

where $H(k, z)$ and $G(k, z)$ are the Fourier transforms of $h(x, z)$ and $g(x, z)$ respectively, and the Kronecker Delta has its usual meaning.

Substituting Eq. 19 into Eq. 15 yields

$$
\begin{aligned}
& -\frac{k}{2 \pi} \int_{-\infty}^{\infty} k^{\prime \prime \prime} \sigma_{P}\left(k-k^{\prime \prime \prime}, z\right) \phi\left(k^{\prime \prime \prime}, z\right) \mathrm{d} k^{\prime \prime \prime} \\
& =-k^{2} \sigma_{P}^{\infty}(z) \phi(k, z) \\
& \quad-\frac{\sigma_{P}^{\infty}(z)}{2 \pi} \int_{-\infty}^{\infty} k k^{\prime \prime \prime} H\left(k-k^{\prime \prime \prime}, z\right) \phi\left(k^{\prime \prime \prime}, z\right) \mathrm{d} k^{\prime \prime \prime}
\end{aligned}
$$

while substituting Eq. 18 into Eq. 16 gives

$$
\begin{aligned}
\frac{1}{2 \pi} \int_{-\infty}^{\infty} \sigma_{\|}\left(k-k^{\prime \prime \prime}, z\right) \frac{\partial^{2} \phi\left(k^{\prime \prime \prime}, z\right)}{\partial z^{2}} \mathrm{~d} k^{\prime \prime \prime} \\
\quad+\frac{1}{2 \pi} \int_{-\infty}^{\infty} \frac{\partial \sigma_{\|}\left(k-k^{\prime \prime \prime}, z\right)}{\partial z} \frac{\partial \phi\left(k^{\prime \prime \prime}, z\right)}{\partial z} \mathrm{~d} k^{\prime \prime \prime} \\
=\sigma_{\|}^{\infty}(z) \frac{\partial^{2} \phi(k, z)}{\partial z^{2}}+\frac{\partial \sigma_{\|}^{\infty}(z)}{\partial z} \frac{\partial \phi(k, z)}{\partial z} \\
\quad+\frac{\sigma_{\|}^{\infty}(z)}{2 \pi} \int_{-\infty}^{\infty} G\left(k-k^{\prime \prime \prime}, z\right) \frac{\partial^{2} \phi\left(k^{\prime \prime \prime}, z\right)}{\partial z^{2}} \mathrm{~d} k^{\prime \prime \prime} \\
\quad+\frac{1}{2 \pi} \int_{-\infty}^{\infty} \frac{\partial}{\partial z}\left(\sigma_{\|}^{\infty}(z) G\left(k-k^{\prime \prime \prime}, z\right)\right) \frac{\partial \phi\left(k^{\prime \prime \prime}, z\right)}{\partial z} \mathrm{~d} k^{\prime \prime \prime}
\end{aligned}
$$

Therefore, the $x$ Fourier transform of Eq. 7 may be written as

$$
\begin{gathered}
-k^{2} \sigma_{P}^{\infty}(z) \phi(k, z)+\sigma_{\|}^{\infty}(z) \frac{\partial^{2} \phi(k, z)}{\partial z^{2}}+\frac{\partial \sigma_{\|}^{\infty}(z)}{\partial z} \frac{\partial \phi(k, z)}{\partial z} \\
=i k E_{x}^{0} \sigma_{P}^{\infty}(z) H(k, z) \\
+\frac{\sigma_{P}^{\infty}(z)}{2 \pi} \int_{-\infty}^{+\infty} k k^{\prime} H\left(k-k^{\prime}, z\right) \phi\left(k^{\prime}, z\right) \mathrm{d} k^{\prime} \\
-\frac{\sigma_{\|}^{\infty}(z)}{2 \pi} \int_{-\infty}^{+\infty} G\left(k-k^{\prime}, z\right) \frac{\partial^{2} \phi\left(k^{\prime}, z\right)}{\partial z^{2}} \mathrm{~d} k^{\prime} \\
-\frac{1}{2 \pi} \int_{-\infty}^{+\infty} \frac{\partial}{\partial z}\left(\sigma_{\|}^{\infty}(z) G\left(k-k^{\prime}, z\right)\right) \frac{\partial \phi\left(k^{\prime}, z\right)}{\partial z} \mathrm{~d} k^{\prime}
\end{gathered}
$$

We note that $k^{\prime \prime \prime}$ and $\mathrm{d} k^{\prime \prime \prime}$ in Eqs. 20 and 21 are dummy variables and can be changed to $k^{\prime}$ and $\mathrm{d} k^{\prime}$ without any loss of generality.

\subsection{Using symmetry to speed up the calculations}

For convenience, the latitudinal precipitation pattern is chosen to be symmetric in our model. More specifically, we assume that the functions $g$ and $h$ of Eqs. 10 and 11 are even, that is to say that they are symmetric about $x=0$. This choice of pattern does not present a problem as long as we choose our boundaries to be sufficiently far from the edge of the arc and as long as the arc itself is wide when compared to the scale length of the gradients $(10 \mathrm{~km}$ compared to $100 \mathrm{~m})$. This means that the Fourier transforms of $g$ and $h, G$ and $H$, are real and symmetric in $k$. Using the fact that, for this particular problem, $\phi(x, z)$ is anti-symmetric in $x$ and that $\phi(k, z)$ is consequently anti-symmetric in $k$, we only need to perform the integrations on the right-hand side of Eq. 22 over positive values of $k^{\prime}$. However, before this integration can be performed, the negative $k^{\prime}$ interval must be expressed in terms of the results obtained for 
the positive $k^{\prime}$ s. As a result, upon turning the integrations into summations, Eq. 22 becomes

$$
\begin{aligned}
- & k^{2} \sigma_{P}^{\infty}(z)\left[1+\frac{\Delta_{k}}{2 \pi}(H(0, z)+H(2 k, z))\right] \phi(k, z) \\
+ & \sigma_{\|}^{\infty}(z)\left[1+\frac{\Delta_{k}}{2 \pi}(G(0, z)-G(2 k, z))\right] \frac{\partial^{2} \phi(k, z)}{\partial z^{2}} \\
+ & {\left[\frac{\partial \sigma_{\|}^{\infty}(z)}{\partial z}+\frac{\Delta_{k}}{2 \pi} \frac{\partial}{\partial z}\left(\sigma_{\|}^{\infty}(z)(G(0, z)-G(2 k, z))\right)\right] } \\
\times & \frac{\partial \phi(k, z)}{\partial z}=i E_{x}^{0} k \sigma_{P}^{\infty}(z) H(k, z) \\
& +k \sigma_{P}^{\infty}(z) \frac{\Delta_{k}}{2 \pi} \sum_{k^{\prime} \neq k} k^{\prime}\left[H\left(k+k^{\prime}, z\right)+H\left(k-k^{\prime}, z\right)\right] \phi\left(k^{\prime}, z\right) \\
& -k \sigma_{\|}^{\infty}(z) \frac{\Delta_{k}}{2 \pi} \sum_{k^{\prime} \neq k}\left[G\left(k-k^{\prime}, z\right)-G\left(k+k^{\prime}, z\right)\right] \frac{\partial^{2} \phi\left(k^{\prime}, z\right)}{\partial z^{2}} \\
& -\frac{\Delta_{k}}{2 \pi} \sum_{k^{\prime} \neq k} \frac{\partial}{\partial z}\left[\sigma _ { \| } ^ { \infty } ( z ) \left(G\left(k-k^{\prime}, z\right)\right.\right. \\
& -G\left(k+k^{\prime}>0\right. \\
\left.\left.\left.k^{\prime}, z\right)\right)\right] & \frac{\partial \phi\left(k^{\prime}, z\right)}{\partial z}
\end{aligned}
$$

where $\Delta_{k}$ is the $k$ interval chosen to change the integration into a summation $\left(\Delta_{k}\right.$ replaces the term $\left.\mathrm{d} k^{\prime}\right)$. Equation 23 is used to find the electrostatic potential $\phi(k, z)$ for $k>0$. The potential for negative values of $k$ are then immediately obtained by symmetry.

\subsection{Boundary conditions}

Having described the simplified electrostatic equation as a function of wavenumber, $k$, and altitude, $z$, we must also apply the appropriate boundary conditions before we can solve for the electrostatic potential, $\phi(x, z)$, using Eq. 23.

The first boundary condition is a zero current requirement at the lower boundary, namely,

$J_{\|}\left(x, z_{\min }\right)=0$

where $z_{\min }$ in our calculations was chosen to be at $50 \mathrm{~km}$ altitude. At altitudes lower than $100 \mathrm{~km}$ the collision frequency begins to increase dramatically and consequently, the parallel conductivity decreases. It is therefore quite safe to assume that at a low enough boundary (we used $50 \mathrm{~km}$ ) we can neglect any parallel current.

The lateral boundary conditions are more problematic in the sense that, with the pseudo-spectral method, they can only be applied at large distances from the precipitating region. They are

$\delta E_{\perp}\left(-x_{\max }, z\right)=\delta E_{\perp}\left(x_{\max }, z\right)=0$

where $\delta E_{\perp}\left( \pm x_{\max }, z\right)$ are the perturbed horizontal electric fields due to the gradients in the Pedersen conductivity. As far as the electrostatic potential is concerned, these conditions imply that $\phi$ must approach a constant value, i.e.,

$\phi\left(-x_{\max }, z\right)=\phi\left(x_{\max }, z\right)=\mathrm{constant}$

where $\pm x_{\max }$ are the horizontal extent of our simulation region. The distance $\pm x_{\max }$ was therefore chosen so as to be sufficiently far from the source region (in our simulations we used $x_{\max }=10 \mathrm{~km}$ ). For simplicity the constant in Eq. 26 was chosen to be 0 .

For our final boundary condition we used

$E_{\|}\left(x, z_{\max }\right)=0$

This last boundary condition is also problematic and must, once again be taken very far from the source region. If taken too close to the ionosphere it forces the high altitude electric field to be a function of horizontal position. This is not consistent with our model since the horizontal conductivities become negligible at high altitudes while the parallel conductivity is simultaneously large. This means that changes in the shape of equipotentials are extremely slow as the altitude increases. This being stated, our main concern is not so much that the upper boundary condition be obeyed perfectly, but rather that it does not influence the ionospheric solutions. This is what dictates our choice of the location of the upper boundary condition. We have found that by the time we reach about $3000 \mathrm{~km}$ altitude the effect of the boundary condition on the ionospheric solution is indeed minimal. Therefore, we picked our upper value of $z$, namely, $z_{\max }$ to be at $3000 \mathrm{~km}$ altitude. As for the value assigned to the potential at the upper boundary we chose it to be the same as the lateral boundary conditions, namely, an equipotential of zero.

\subsection{The solution of the electrostatic equation}

The partial $z$-derivatives in Eq. 23 have been expressed as centered differences. We covered a vertical range from $z=50$ to $3000 \mathrm{~km}$ using a non-uniform grid spacing. The vertical cell size varies according to the height, from $5 \mathrm{~km}$ at $z \leq 400 \mathrm{~km}$ to $40 \mathrm{~km}$ at $z \geq 1600 \mathrm{~km}$. A Lagrangian interpolation scheme is used to compute the derivatives at the altitude where the step size is changing. Using a variable step size in the vertical allows us to concentrate on the region where the changes are taking place while ensuring that our upper boundary is sufficiently far.

For the cases presented here we chose the horizontal dimension to be $20 \mathrm{~km}$ wide with a precipitating region $10 \mathrm{~km}$ wide. Recall that our main interest is not the width of the precipitation region but rather the sharpness of its boundary and the ensuing large gradients in the Pedersen conductivity. This requires that the horizontal dimension be covered sufficiently well. Therefore, the total number of wavenumbers used in the $x$ dimension was set at 1024, effectively giving a horizontal resolution of $\sim 20 \mathrm{~m}$ for the runs that we will present. Note that the horizontal resolution may be increased by simply increasing the total number of wavenumbers 
used in the calculation. However, this obviously increases the total simulation time.

The use of centered differences in $z$ produces a tridiagonal matrix that can readily be inverted using standard techniques. This means that the $z$ part of the problem can be inverted in a single step, without the need for an iterative method. However, the inversion has to be performed for each value of $k$. Furthermore, the coupling between the different $k$ values in Eq. 23 forces us to use an iterative method for the Fourier part of the problem. Specifically, we used the solution from previous iterations on the right-hand side of Eq. 23 to compute the improved iterate on the left-hand side of Eq. 23.

We also used a relaxation technique to speed up the rate of convergence. To be more specific, let us introduce superscripts $n$ and $n-1$ for the $n^{\text {th }}$ and $n^{\text {th }}-1$ iteration, respectively, and also a relaxation parameter $\omega$. We calculated the $n^{\text {th }}+1$ iterate on the left-hand side of Eq. 23 by replacing the value of $\phi^{(n)}\left(k^{\prime}, z\right)$ in the summations of the right-hand side of Eq. 23 viz

$\phi^{(n)}\left(k^{\prime}, z\right) \rightarrow \phi^{(n)}\left(k^{\prime}, z\right)+\omega\left(\phi^{(n)}\left(k^{\prime}, z\right)-\phi^{(n-1)}\left(k^{\prime}, z\right)\right)$

where $\phi^{(n-1)}\left(k^{\prime}, z\right)$ is the potential at the $n^{\text {th }}-1$ iteration and $\phi^{(n)}\left(k^{\prime}, z\right)$ is the improved solution for the potential.

The relaxation parameter in our model was held constant at 0.5 with the exception that for $n=1$ we had to use $\omega=0$. The values of $\phi^{(1)}(k, z)$ were then calculated. The new values of $\phi(k, z)$ were then inserted back in Eq. 28 to calculate the $\phi^{(n)}\left(k^{\prime}, z\right) \mathrm{s}$ which were used in the summations on the right-hand side of Eq. 23 for the next iteration. This procedure was then repeated until the $\phi(k, z) \mathrm{s}$ had converged to the desired relative error. The relative error calculation, $\epsilon$, is evaluated from

$\epsilon=\frac{1}{N_{k}} \frac{1}{N_{z}} \sum_{k}^{N_{k}} \sum_{z}^{N_{z}}\left|\frac{\phi^{(n+1)}(k, z)-\phi^{(n)}(k, z)}{\phi^{(n+1)}(k, z)}\right|$

where $N_{k}$ is the total number of wavenumbers used to compute the electric potential, $\phi(k, z), N_{z}$ is the total number of altitude steps. We iterate until $\epsilon$ is smaller or equal to the preset maximum tolerable relative error chosen for the simulation.

Finally we recall that once the electric potential, $\phi(k, z)$ has been evaluated it is straightforward to obtain $\phi(x, z)$ by performing the inverse Fourier transform to obtain $\phi$ itself. From $\phi$ we can then easily determine the electric fields and current densities.

\section{The coupled model}

As stated in Sect. 2, the electrostatic model is coupled to a comprehensive time-dependent transport model. The transport model will be referred to as TRANSCAR while the electrostatic model will be referred to as ELECTRO from here on.

TRANSCAR is a one-dimensional program that models the terrestrial ionosphere in the altitude range $100-3000 \mathrm{~km}$, in the direction parallel to the Earth's magnetic field. It contains two distinct parts. The first part consists of a fluid description of the ionosphere and is based on an 8-moment formulation (Blelly and Schunk, 1993). It describes six thermal ion species $\left(\mathrm{O}^{+}, \mathrm{H}^{+}, \mathrm{N}^{+}, \mathrm{N}_{2}^{+}, \mathrm{O}_{2}^{+}\right.$and $\left.\mathrm{NO}^{+}\right)$as well as the thermal electrons. It computes the temporal evolution of the concentrations, the field aligned velocities, the average temperature and the field-aligned heat flow for each ionic species as well as for the electrons.

The second part of TRANSCAR is a kinetic transport model of energetic electrons which accounts for the precipitation of electrons originating from the magnetosphere and for photoelectrons resulting from the solar photon actions on the neutral atmosphere (Lilensten et al., 1988; Lummerzheim and Lilensten, 1994; Blelly et al., 1996). The kinetic model provides the ion production rates and electron heating source for the fluid model.

The equations used in the fluid formulation may be found in Blelly and Schunk (1993). We shall only discuss here the equations that we had to modify. For a complete description of TRANSCAR see Blelly et al. (1995).

\subsection{The continuity equation}

The time-dependent continuity equation for species $i$ may be written as,

$\frac{\partial n_{i}}{\partial t}+\frac{1}{A} \frac{\partial}{\partial r}\left(A n_{i} u_{i}\right)=P_{i}-L_{i}$

where $r$ represents the path along the magnetic field line, $A$ corresponds to the perpendicular cross section on the magnetic flux tube and, $P_{i}$ and $L_{i}$ are the chemical production and loss terms, respectively, $n_{i}$ is the number density of species $i$ and $u_{i}$ is the field-aligned velocity of species $i$.

The chemical reactions and the ionization rates that are used in TRANSCAR may be found in Schunk and Nagy (1980), Rees (1989) or Diloy et al. (1996). It should be noted that some of the chemical reactions are strongly temperature dependent. This could lead to some interesting feedback when the electrodynamics is included with the transport models. In that regard, the reaction rates involving $\mathrm{O}^{+}$have been replaced by the rates obtained by St.-Maurice and Laneville (1998). These reaction rates are similar to those that are normally used in ionospheric work except for the fact that they cover a far greater temperature range. We have to use these new rates because of the very large electric fields that we may want to use during the course of our simulations. With the existence of very large fields, the heating due to friction with the neutrals could raise the effective temperature well above the temperatures posted for the reaction rates that are normally used and which had originally been obtained by St.-Maurice and Torr (1978).

The electron density is easily evaluated directly using charge neutrality, namely,

$n_{e}=\sum_{i} n_{i}$ 


\subsection{The electron velocity}

We obtain the electron velocity from the parallel currents, viz

$J_{\|}=q\left\{\sum_{i} n_{i} u_{i}-n_{e} u_{e}-F_{\text {supra }}\right\}$

where $u_{i, e}$ is the parallel velocity of species $i$, electrons, $n_{i, e}$ is the number density of species $i$, electrons and $F_{\text {supra }}$ is the suprathermal electron flux due to electron precipitation. The suprathermal electron flux is obtained directly from the kinetic part of the model.

Therefore, the ambipolar flow is obtained from

$n_{e} u_{e}=\sum_{i} n_{i} u_{i}-F_{\text {supra }}-\frac{J_{\|}}{q}$

The last term is an important element of the coupling between TRANSCAR and ELECTRO and takes considerable importance in the presence of sharp horizontal gradients in the plasma density and hence, in the Pedersen conductivity.

\subsection{The energy equation}

Detailed expressions for the energy transport equations may be found in Blelly and Schunk (1993) and Blelly et al. (1996). In the present context, we simply wish to emphasize that for each species the frictional heating source connected to the $\mathbf{E} \times \mathbf{B}$ drift has been included in TRANSCAR. The expression for the heating rate may be found in many places (e.g., Schunk and Walker, 1971; Robineau et al., 1996) and it reads,

$Q_{E}^{s}=\frac{n_{s} e_{s}^{2} v_{s} E_{\perp}^{2}}{m_{s}\left(v_{s}^{2}+\Omega_{s}^{2}\right)}$

where $E_{\perp}$ is the perpendicular convective electric field amplitude, $\Omega_{s}=e_{s} B / m_{s}$ is the gyrofrequency of species $s, \quad v_{s}=\sum_{t} \frac{m_{s}}{m_{s}+m_{t}} v_{s t}$ is the total momentum transfer frequency between species $s$ and $t, e_{s}$ is the charge of species $s, m_{s}$ is the mass of species $s$ and $Q_{E}^{s}$ is the heating rate of species $s$ due to the perpendicular electric field.

As far as the electrons are concerned, the heating term $Q_{E}^{e}$ is usually not important for small to modest electric field strengths. However, for some of the very large electric fields that we have considered this is no longer the case. With this in mind, we also note from Eq. 34 that if $E_{\perp}$ is structured, the heating rate and associated temperatures will reflect that structure.

\section{Particulars of the coupling between TRANSCAR and ELECTRO}

As stated at the beginning of the last section, TRANSCAR is a one-dimensional time-dependent model while ELECTRO is two-dimensional and time-independent because of the very fast time scales over which the $\nabla \cdot \mathbf{J}=0$ condition is satisfied (Sect. 3). In order to couple the two models, TRANSCAR was modified effectively to become two-dimensional.

The first step was to specify the number of field lines to be used in the simulation. The number of field lines is arbitrary but we require a sufficient number to adequately describe the gradients on the edges of the precipitation region. TRANSCAR initializes each field line for the number densities, temperatures, velocities and heat flows of each ionic species and electrons. Once each field line has been initialized we impose a precipitation pattern onto the constant background.

The latitudinal pattern for the precipitation flux has been described by the functional

$f(x)=\frac{\arctan [a(x+\Delta)]-\arctan [a(x-\Delta)]}{2 \arctan (a \Delta)}$

where $x$ is the horizontal position in $\mathrm{km}$ and, $a$ is used to specify the steepness of the gradient in $f(x)$ and where $\Delta$ is used to specify the latitudinal position of the maximum horizontal gradient. The function $f(x)$ is normalized so that the maximum value of the precipitation factor is unity at the center of the arc, while far from the center of the arc i.e., $x \rightarrow \pm \infty$, the factor approaches 0 implying no precipitation far from the center. In Fig. 1 we present a plot of Eq. 35 with $a=5$, 10 and $20 \mathrm{~km}^{-1}$ and $\Delta=5 \mathrm{~km}$. We observe that as the value of $a$ increases, the steepness of the function near $x=\Delta$ also increases. Our functional, Eq. 35, was chosen to be symmetric about $x=0$ so as to enable us to take advantage of the symmetry of the problem, as discussed in Sect. 3.

Once Eq. 35 is used to describe the latitudinal precipitation pattern in our simulations, TRANSCAR computes the time-evolution of the concentrations, temperatures, and field-aligned velocity for each species on each individual field line independently. The concentrations, temperatures and the imposed background electric field are then provided to ELECTRO. The

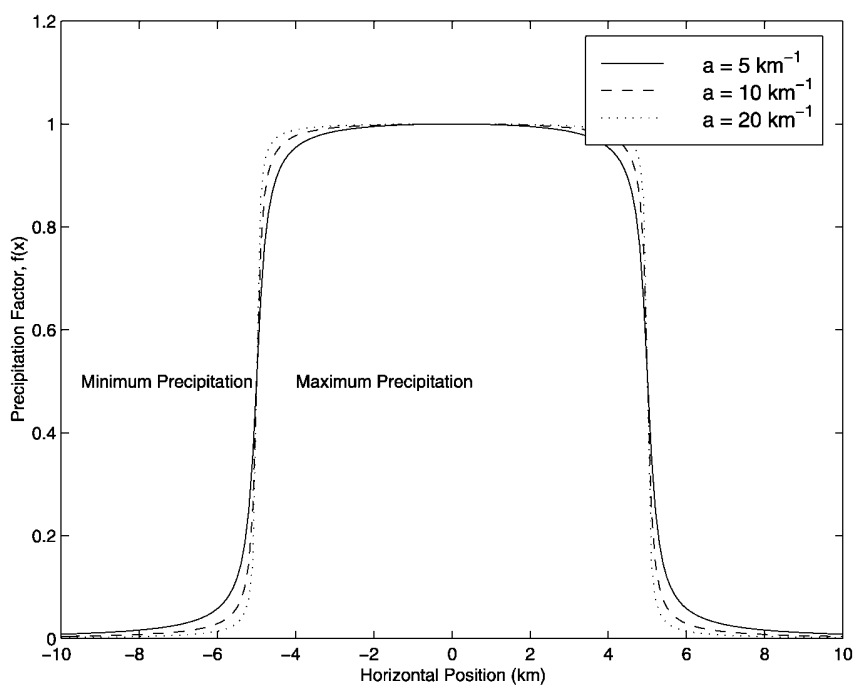

Fig. 1. Function $f(x)$ used to model the latitudinal pattern of the precipitation. The horizontal position denotes the latitudinal distance from the center of the precipitation pattern 
electrostatic model proceeds to compute the conductivities, $\sigma_{\|}(x, z)$ and $\sigma_{P}(x, z)$ on each field line for the particular time. We recall that the number of field lines chosen is arbitrary as long as the gradient in the Pedersen conductivity is covered adequately. To obtain the conductivities on the field lines that are not simulated by TRANSCAR a linear interpolation scheme was used.

The uniform background conductivities, $\sigma_{\|}^{\infty}(z)$ and $\sigma_{P}^{\infty}(z)$ are computed using the concentrations and temperatures of the first field line where no precipitation is present. The perturbations in the conductivities, $\sigma_{P}(x, z)$ and $\sigma_{\|}(x, z)$, are computed using Eqs. 10 and 11 , respectively.

The Fourier transform, $H(k, z)$ and $G(k, z)$, are determined numerically and the electric potential is found via Eq. 23. Once the electric potential is determined, the perturbed electric fields and the field-aligned current density are computed and provided to TRANSCAR. The transport model then proceeds to compute the plasma parameters for the next time step, using the electrodynamics provided by ELECTRO. The whole process can continue indefinitely. Of particular interest, however, is the state of the ionosphere after the initial rise in conductivities a few tens of seconds after the onset of precipitation. In this initial presentation we focus on this kind of time scale. There are of course longer time scales involved as well, in association with the response to pressure gradients caused by heating, for instance. These longer time scales will not be considered here because they modify the current densities by a much smaller amount than what is found in the first few tens of seconds following the onset of precipitation. A second point to keep in mind is that the model calculations cease to be valid if the medium starts to evolve short-scale irregularities triggered, for example, by two-stream instabilities between ions and electrons. In that case the short-scale structures cannot be modeled with our transport equations, and the best that can be done would be to add anomalous transport (or wave-induced) equations to the system to take into account the presence of these new nonlinearities. This is not being done at this point in time. In fact one of our goals is to establish if the situations described by our model can indeed be the cause for short-scale irregularities through some appropriate instability mechanism.

\section{First results}

In this section, we present the results of two numerical simulations using TRANSCAR and ELECTRO one hundred seconds after introducing electron precipitation into the system. The first involves a simulation without any coupling between the two models. This run will serve as a benchmark for the second simulation, which has the coupling.

In Fig. 2 we present a plot of the initial electron precipitation spectral flux that was used in both simulations. This spectral flux was chosen because it repre-

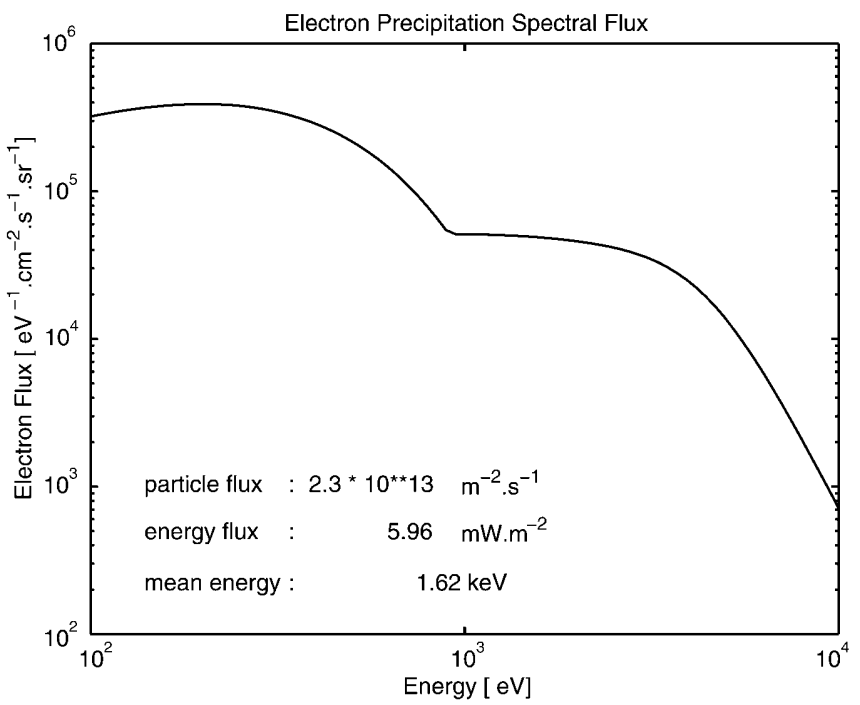

Fig. 2. Precipitating electron spectrum used in TRANSCAR for the runs shown in Figs. 3 to 6

sents a realistic spectral flux (see Lathuillere et al., 1997). The energy flux is $5.96 \mathrm{~mW} / \mathrm{m}^{2}$ and the mean energy of the precipitating electron is $1.62 \mathrm{keV}$. We also note that there are a significant number of electrons in the high energy range, i.e., energies in excess of $1 \mathrm{keV}$, that can penetrate deep into the ionosphere and reach the $E$-region before they are effectively stopped.

The ambient convective electric field was chosen in both cases to be $110 \mathrm{mV} / \mathrm{m}$ and to be oriented exactly along the $x$ direction (no arc motion). As discussed in the Appendix, the results should be representative of situations with drifting arcs in the presence of strong ambient electric fields, as long as the $\left|E_{y}^{0}\right| \ll\left|E_{x}^{0}\right|$. The parameters $a$ and $\Delta$ in Eq. 35 were chosen to be $10 \mathrm{~km}^{-1}$ and $5 \mathrm{~km}$ respectively. Our choices of $a$ and $\Delta$ correspond to an arc $\sim 10 \mathrm{~km}$ wide having a gradient scale length of $\sim 200 \mathrm{~m}$.

\subsection{Case 1 - uncoupled}

In Fig. 3 we present the output from TRANSCAR for the uncoupled run. By "uncoupled" we mean the following: we ran TRANSCAR for $100 \mathrm{~s}$ after the onset of precipitation and calculated the conductivities resulting from this run. We then fed these conductivities into ELECTRO and computed the resulting electric potential, fields and current densities, but did not feed this information back into TRANSCAR.

The top two panels of Fig. 3 correspond to the electron and $\mathrm{O}^{+}$density profiles as a function of horizontal position and altitude. The middle two panels are the electron and $\mathrm{O}^{+}$temperature profiles while the lower two panels correspond to the electron and $\mathrm{O}^{+}$ field-aligned velocities, both in $\mathrm{m} / \mathrm{s}$.

In the electron density panel, we observe a density enhancement at $\sim 120 \mathrm{~km}$ altitude over the region of precipitating electrons. This enhancement is consistent with what we would expect from the spectral flux shown 

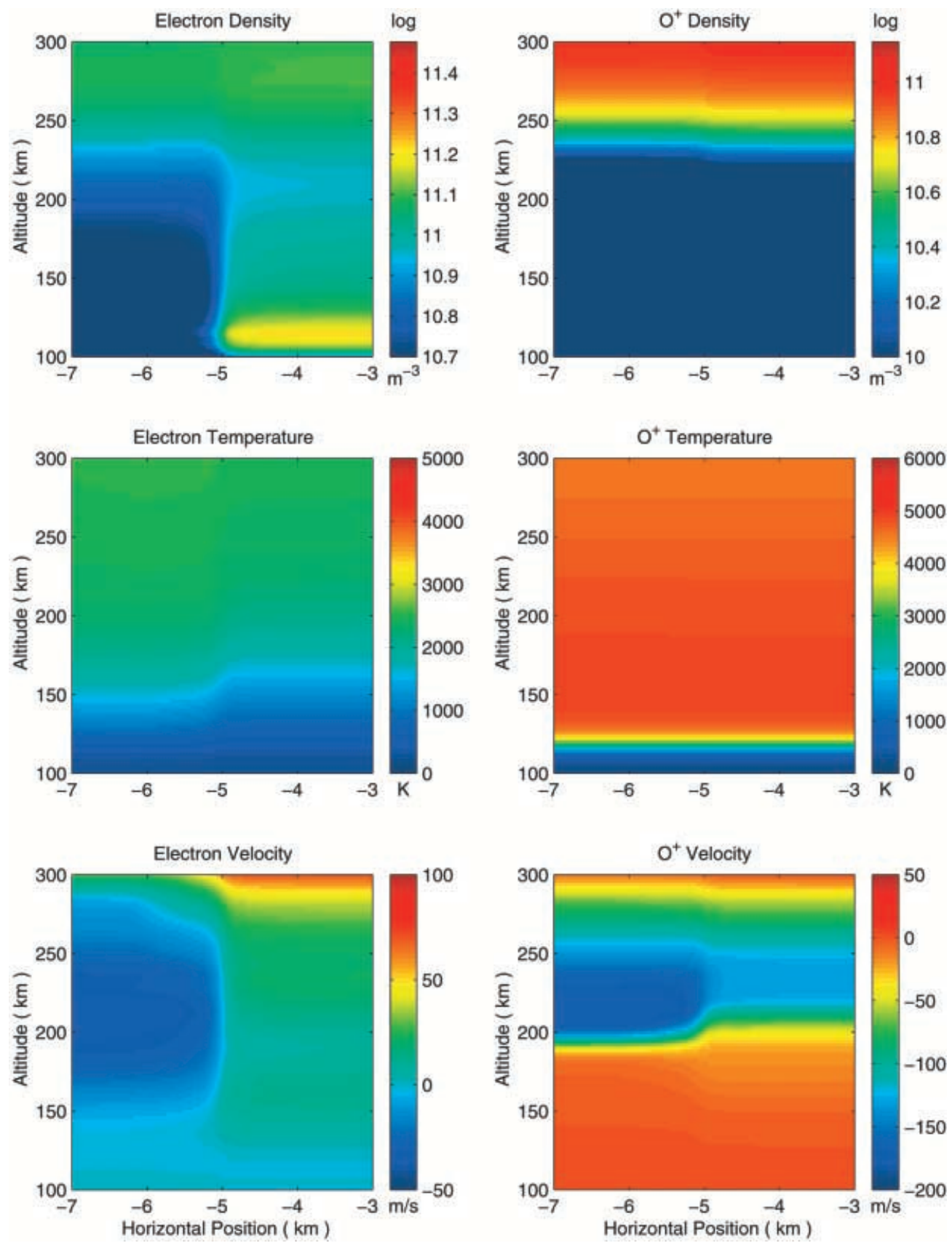

Fig. 3. Output from TRAN-

SCAR one hundred seconds after the onset of precipitation for a $110 \mathrm{mV} / \mathrm{m}$ background electric field. In this case TRANSCAR is solved first for $100 \mathrm{sec}$, after which the potential is obtained from the electrodynamics code. For the purpose of comparing with the self-consistent cases shown in later figures no other form of coupling between ELECTRO and TRANSCAR is performed. Top panels: $n_{e}$ and $n_{\mathrm{O}^{+}}$; middle panels: $T_{e}$ and $T_{\mathrm{O}^{+}}$; bottom panels: $v_{e \|}$ and $v_{\mathrm{O}^{+}}$ in Fig. 2. On the other hand, the $\mathrm{O}^{+}$density remains fairly constant throughout the region.

In the electron temperature profile, we observe a slight decrease in the electron temperature within the precipitation region. This is due to the larger heat capacity of the field lines with enhanced electron densities. By contrast, the $\mathrm{O}^{+}$temperature is elevated throughout the region both inside and outside the arc. The elevated temperature is consistent with the frictional heating caused by the convective electric field (see Eq. 34) and also with the fact that it only takes one collision time (a few seconds at most) for the ion temperature to increase through friction in the $\mathrm{E}$ and $\mathrm{F}$ region. Similar effects can be seen for other ionic species even though they are not presented here.
In Fig. 4 we present the parameters of electrodynamical interest based on the results presented in Fig. 3 and on the calculations of the potential by ELECTRO, using the TRANSCAR output as input. The top two panels describe the Pedersen and parallel conductivities, the middle panels are the electrostatic potential and current density and the bottom panels are the perturbed perpendicular and parallel electric fields.

In the Pedersen conductivity panel, we observe a significant enhancement at an altitude of $\sim 120 \mathrm{~km}$ throughout the precipitation region. This enhancement coincides with the electron density enhancement seen in Fig. 3 (top left panel). The horizontal gradient in the Pedersen conductivity results in a field-aligned current density on the order of $320 \mu \mathrm{A} / \mathrm{m}^{2}$ and is localized in a 

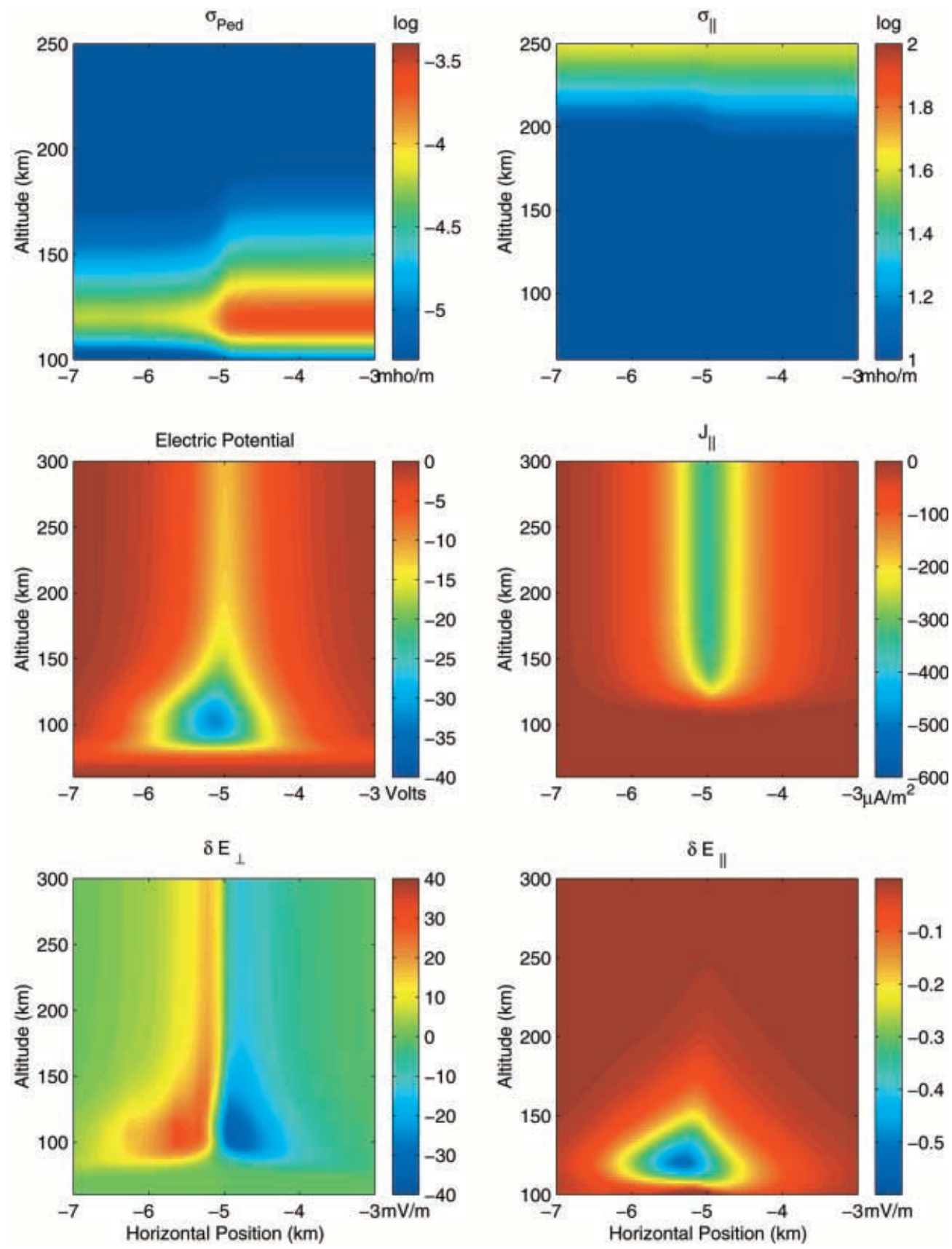

Fig. 4. Output from the electrodynamics part of the model for the case shown in Fig. 3. Top panels: $\sigma_{P}$ and $\sigma_{\|}$; middle panels: $\phi(x, z)$ and $J_{\|} ;$bottom panels: $\delta E_{\perp}$ and $\delta E_{\|}$ narrow region $200 \mathrm{~m}$ wide. The magnitude of the current density is of the same order of magnitude as those obtained in the study of St.-Maurice et al. (1996) and is quite a bit larger than the $1-5 \mu \mathrm{A} / \mathrm{m}^{2}$ auroral average.

As far as the perpendicular electric field profile is concerned, we note the presence of a velocity shear of $1-2 \mathrm{~m} / \mathrm{sec}$ per meter. This velocity shear is of the same order of magnitude as those obtained by St.-Maurice et al. (1996).

One final point should be remembered from this first run: even though the coupling between the two models in this case was neglected, we see from the electrodynamical response in Fig. 4 that gradients in the Pedersen conductivity already play quite an important role in the electrodynamics just $100 \mathrm{sec}$ after the onset of precipitation.

\subsection{Case 2 - coupled}

We present the results produced by the fully coupled model in Figs. 5 and 6. These figures are derived from the same input parameters as Figs. 3 and 4 except for the fact that self-consistent coupling effects are now taken into account as the transport model was marching forward in time. When comparing Figs. 3 and 4 to Figs. 5 and 6 we immediately observe many significant differences. These differences show that the coupling between the composition, energetics and electrodynamics is indeed an important piece of the picture.

To start with, there is a significant difference in the electron densities seen in Figs. 3 and 5. The electron density of Fig. 5 is considerably enhanced in a region located just inside the arc, particularly at an altitude 

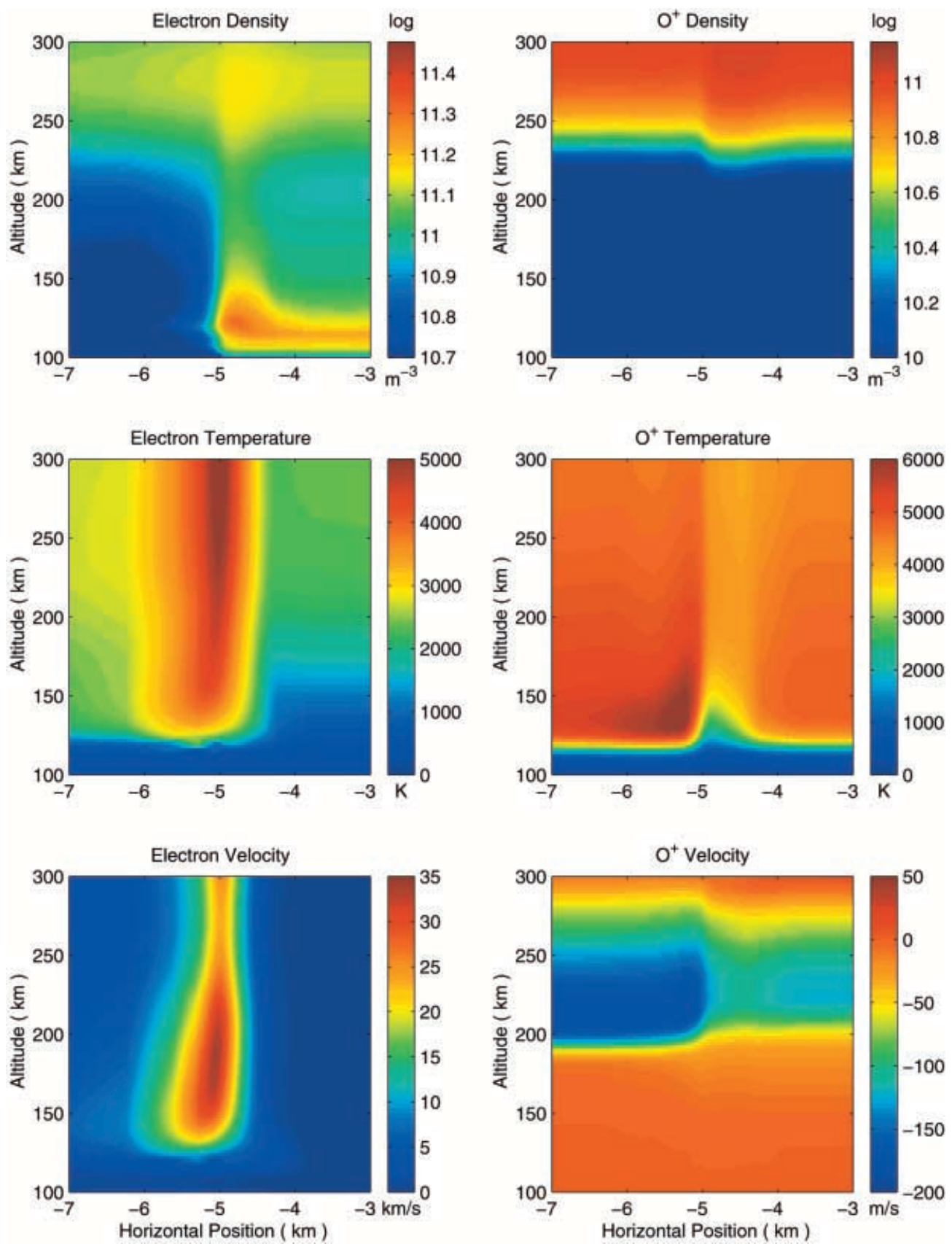

Fig. 5. Same as in Fig. 3 but with the inclusion of self-consistent coupling between transport and electrodynamics $\sim 120 \mathrm{~km}$ (although all altitudes are actually enhanced). The change in the electron density is a good example of the kinds of nonlinear feedback that we were looking for when developing our coupled time-dependent model. Specifically, the electron density increase is associated with a dramatic increase in the electron temperature on the edge of the arc. That temperature is in turn produced by the large parallel current densities that could already be seen in Fig. 4. The reason for the increased electron density is simply that the recombination rates of the molecular ions are considerably smaller when the electron temperature is enhanced (see Eq. 12). The reason for the relatively large increase at $120 \mathrm{~km}$ is that this is a region with a considerable molecular ion production rate through precipitation.
The feedback on the electron density is important because it in turns affects the parallel currents that started the electron heating. That is to say: the enhancement in $n_{e}$ coincides with an enhancement in the Pedersen conductivity which can be seen to happen in the same location in Fig. 6, top left panel. More importantly, one can see that the horizontal gradient in the Pedersen conductivity increases dramatically on the edge of the arc as a result. As seen when discussing Eq. 7, a steepened conductivity gradient in turn can (and, in this case, does) lead to the intensification of parallel current densities which now reach $\sim 500 \mu \mathrm{A} / \mathrm{m}^{2}$. This represents a $50 \%$ increase in magnitude when compared to the uncoupled case after $100 \mathrm{~s}$ of simulation time. Note that the increase in parallel current 

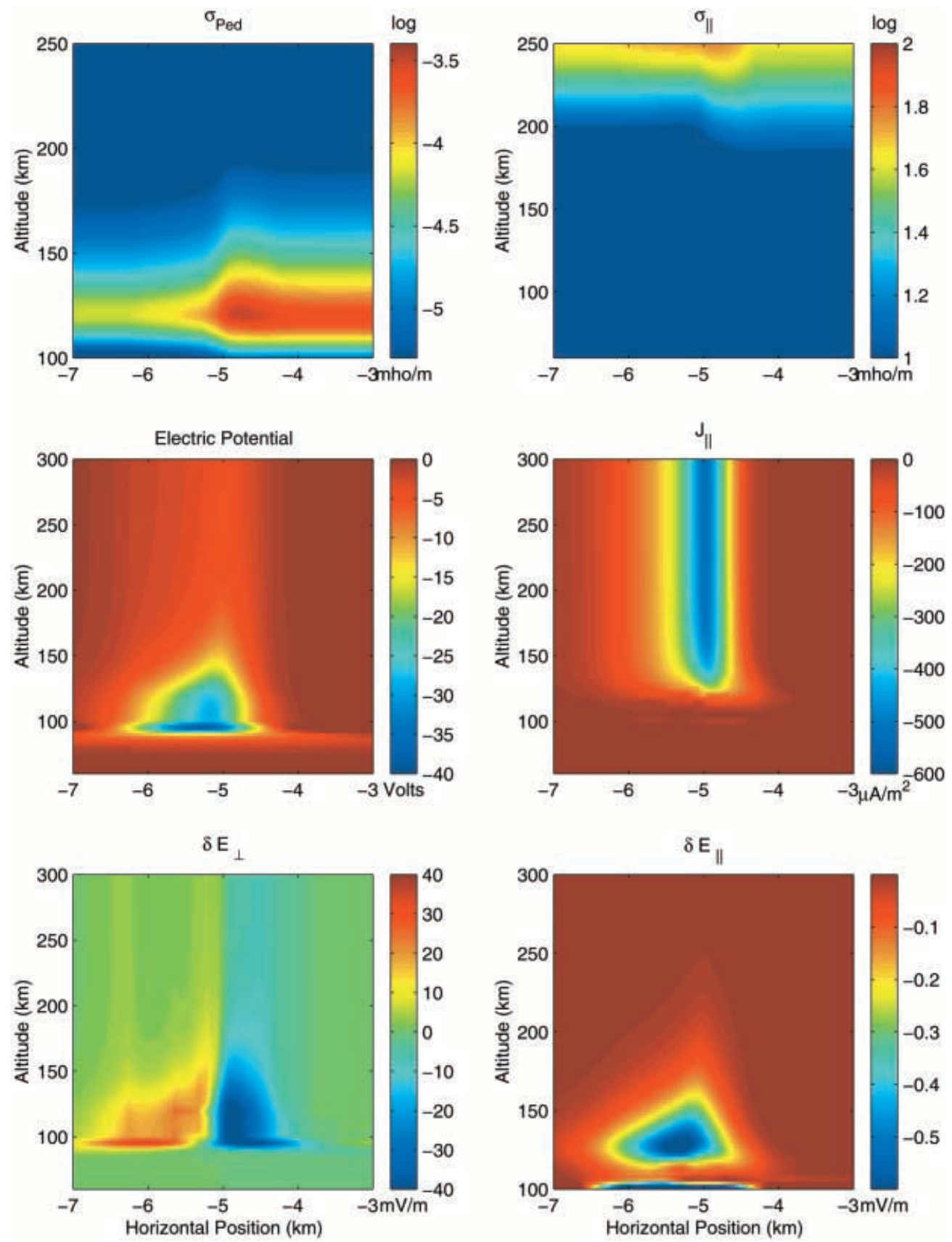

Fig. 6. Same as in Fig. 4 but with the inclusion of self-consistent coupling between transport and electrodynamics densities heats the electrons further, and so on: the effect on the parallel currents is large because of this nonlinear positive feedback process between parallel currents and Pedersen conductivity gradients.

Associated with the large parallel current densities are large field-aligned electron velocities. These velocities were not computed self-consistently in Fig. 3 but should obviously have been calculated (hence the need for self-consistency between the dynamics and the electrodynamics). The difference between the drifts from the uncoupled to the coupled case is of course huge as a result, i.e., $\sim 35 \mathrm{~m} / \mathrm{sec}$ in Fig. 3 compared to $\sim 35 \mathrm{~km} / \mathrm{sec}$ in Fig. 5. The latter velocity is incidentally large enough to suggest that the plasma instabilities observed by Rietveld et al. (1991) and Collis et al. (1991) could indeed have been produced by intense parallel current densities of the kinds obtained here. The numbers are still too small by about a factor of 2 at this point for the two-stream instability to be triggered. However, note it is likely that we could increase the current densities just by fine-tuning some of the input parameters. Furthermore, Gavrishchaka et al. (1998) have suggested that shears in the parallel component of the thermal electron velocity could lower the threshold velocity of the twostream instability. Indeed, we observe in Fig. 5, bottom left panel, that our mechanism does produce appreciable parallel velocity shears.

Another interesting contrast between the coupled and uncoupled runs has to do with the differences in the disturbed electric fields that are obtained. For the 
coupled case, in the region of high Pedersen conductivity, the total $E_{\perp}$ near $130 \mathrm{~km}\left(E_{\perp}=E_{0}+\delta E_{\perp}\right)$ decreases to $\sim 60 \mathrm{mV} / \mathrm{m}$ while in the region of low conductivity $E_{\perp}$ increases to $\sim 135 \mathrm{mV} / \mathrm{m}$. This change in $E_{\perp}$ results in a velocity shear of $1-2 \mathrm{~m} / \mathrm{s}$ per $\mathrm{m}$, comparable to the velocity shears of the uncoupled case. However, we should note the presence of an offset of $\sim 10 \mathrm{mV} / \mathrm{m}$ in the disturbed electric field between the two cases. Another noticeable point is that the perpendicular field decreases more rapidly with altitude in the coupled case than in the uncoupled case. This is consistent with the fact that the parallel electric field in the transition region around $130 \mathrm{~km}$ altitude is 3 times larger in the coupled case than in the uncoupled case.

Another obvious effect associated with the coupling is the horizontal structuring in the ion temperatures. This structuring is simply the result of regions of stronger $E_{\perp}$ corresponding to regions of elevated ion temperature resulting from the heating term shown in Eq. 34. Indeed one can show that the ion temperature in the large electric field cases depends roughly quadratically on the electric field strength. A doubling of the electric field from one region to another therefore represents roughly a quadrupling of the ion temperature. The result is the very large $\mathrm{O}^{+}$temperature gradient that can be seen in Fig. 5.

The main results of the coupled case (100 s after the onset of precipitation) can be summarized as follows:

1. Sharp horizontal gradients in the electron density lead to sharp horizontal gradients in the Pedersen conductivity.

2. Sharp gradients in the Pedersen conductivity lead to the creation of very intense field-aligned current densities that are highly localized in space.

3. Intense field-aligned current densities increase the electron temperatures thereby further reducing the molecular recombination.

4. A reduction in the recombination allows the electron density to increase in the presence of electron precipitation. This results in a further enhancement in the gradient of the plasma density and consequently, the Pedersen conductivity.

A major result of our model is therefore the demonstration of the existence of a positive feedback between the plasma density, the electron temperature, the Pedersen conductivity gradients and the resulting current densities. The feedbacks between the electrodynamics, composition and energetics for a given spectral flux and gradient scale length create a perturbed horizontal field that is directed toward the region of enhanced conductivity gradients. Inside the arc, the net electric field decreases while outside the arc the electric field increases. The shear in the electric field implies a shear in the drift velocity and in the case considered here, a shear of magnitude $1-2 \mathrm{~m} / \mathrm{sec}$ per meter is obtained.

When all of this is put together, we get field-aligned currents of $500 \mu \mathrm{A} / \mathrm{m}^{2}$ in a narrow region about $200 \mathrm{~m}$ wide, $100 \mathrm{~s}$ after the onset of precipitation. This current density is $\sim 500$ times larger than what is found on average. However, it is of the same order of magnitude as the localized current densities estimated by Marklund et al. (1982) and those computed in the steady state study by St.-Maurice et al. (1996).

\section{Conclusion and summary}

The primary goal of the present study was to describe a new time-dependent model of the ionospheric dynamics that would simultaneously solve for the transport processes (continuity, momentum, energy, heat flow and precipitation) and the electrodynamics $\left(E_{\perp}, E_{\|}\right.$and $J_{\|}$) following the onset of intense particle precipitation and in the presence of a uniform background electric field. Foremost in our mind has been the notion that horizontal gradients in conductivity could be as small as $10 \mathrm{~m}$ in size, although we have only for the moment considered gradient scales of the order of 100 to $200 \mathrm{~m}$ in size. The main point is that this model offers a first step toward a self-consistent description of short-scale electrodynamical processes in the ionosphere.

We have emphasized that the model consists of three parts. The first part involves a fluid description based on an 8-moment formulation and describes the thermal ionic population as well as the thermal electrons. This part of the model yields the temporal evolution of the concentrations, field-aligned velocities, the average temperatures and field-aligned heat flow for each species along a magnetic field line.

The second part of the model is a kinetic transport model of energetic electrons which accounts for the precipitation of electrons originating from the magnetosphere and for photoelectrons resulting from solar photon action on the neutral atmosphere (Lilensten et al., 1989; Lummerzheim and Lilensten, 1994; Blelly et al., 1996). This part of the model provides the ion production rates and the electron heating source to the fluid model.

The third part of the model is the electrodynamical code which computes the electrostatic potential and the perturbed current densities and the perturbed electric fields. This model is for the moment based on a simple classical Ohm's law and is used to describe the electrostatic potential in a localized region of space. To solve for the electrostatic potential as a function of position, we have introduced a pseudo-spectral representation of the resulting equation. This technique was chosen because it is computationally faster than other simpler techniques based, for instance, on finite difference schemes.

We have presented results from one example of a model run for $100 \mathrm{sec}$ to illustrate the important role played by various plasma feedback mechanisms when we introduce a $100 \mathrm{~m}$ gradient scale in the conductivities through a sharp horizontal cut-off in the precipitating electron flux. We have shown that the feedback terms can enhance the parallel current densities through the creation of hot regions generating larger conductivity gradients and still more intense parallel currents. We also found that parallel electron drifts of the order of $40 \mathrm{~km} / \mathrm{s}$ and probably more can be created in regions of 
elevated electron temperatures, which suggests that radar observations of ion-acoustic instabilities near auroral arcs may indeed be caused by a two-stream instability between ions and electrons.

There are obviously many possible geophysical situations to consider with this model. Some of the most obvious examples that our model can handle include detailed studies of what happens when we vary the width of the gradient scales as well as the width of the arc itself. We also plan to consider changes in the kinds of precipitating electron fluxes and in the background electric fields, including situations in which there is an $\mathbf{E}_{0} \times \mathbf{B}$ drift component perpendicular to the arc itself.

Acknowledgements. This work was supported by a grant from the Natural Sciences Engineering and Research Council of Canada.

Topical Editor M. Lester thanks a Referee for his help in evaluating this paper.

\section{Appendix: why the present modeling approach is appropriate even for drifting arcs}

It may at first sight seem wrong to neglect the fact that, for instance, east-west elongated arcs could have a north-south drift. For instance, the observation of coherent echoes produced along magnetic field lines has been associated with the passing over the radar field of view of either a narrow precipitation structure (e.g., Sedgemore-Schulthess et al., 1999) or of larger structures with possibly sharp gradients (e.g., Collis et al., 1991). We show here, however, that as long as charge neutrality is obeyed (time scales longer than the inverse of the electron plasma frequency, as shown by St.-Maurice et al., 1996), the description used in our work is appropriate in a reference frame moving with the arc, i.e., with the precipitation. Of course, the precipitating electrons must also be assumed to be $\mathbf{E}_{0} \times \mathbf{B}$ drifting for this result to hold. This may not always be the case, and for these situations our model would not apply.

While our proof could be generalized further, we will limit ourselves here to the situation described in the main text, that is: (1) altitudes $100 \mathrm{~km}$ and above, where electrons are simply $\mathbf{E}_{0} \times \mathbf{B}$ drifting; (2) no derivatives in the $y$ direction, implying that the structures are elongated in that direction. For simplicity, we will also neglect variations in $E_{y}^{0}$ as the arc travels. Using these assumptions, the proof starts simply with an analysis of the electron continuity equation in the presence of our elongated structures. With the $E_{y}^{0}$ field and for the $E$ and $F$ regions, this Eq. reads

$\frac{\partial n_{e}}{\partial t}+\frac{E_{y}^{0}}{B} \frac{\partial n_{e}}{\partial x}=P_{e}-L_{e}-\frac{\partial\left(n_{e} V_{e \|}\right)}{\partial z}$

It is important to realize that the production term $P_{e}$ is moving with the precipitating electrons. As long as these precipitating electrons are $\mathbf{E}_{0} \times \mathbf{B}$ drifting, and if the arc is along the $y$ direction, this means that $P_{e}$ is moving at a speed $E_{y}^{0} / B$ in the $x$ direction, just like the bulk electrons and the arc. The chemical loss terms, $L_{e}$, are likewise drifting at the $E_{y}^{0} / B$ speed in the $x$ direction since the electrons (and therefore the ions, through charge neutrality) are drifting in that manner. This means that we can solve for the electron density in a frame of reference moving at $E_{y}^{0} / B$ in the $x$ direction as if the arc and its production and loss terms were not moving at all, as is done in our simulations.

On the other hand, the ion momentum equation does not say that the ions are $\mathbf{E}_{0} \times \mathbf{B}$ drifting, particularly below $130 \mathrm{~km}$. This only means that in order for the ions to follow the electrons, electric fields must be set up at $E$ region heights. These electric fields will be such as to force the ions to drift with the electrons (otherwise charge neutrality would be violated). After a very short time we must therefore have the condition

$\frac{\partial\left(n_{i} V_{i x}\right)}{\partial x}=\frac{E_{y}^{0}}{B} \frac{\partial n_{e}}{\partial x}+\frac{\partial\left(n_{e} V_{e \|}\right)}{\partial z}-\frac{\nabla \cdot \mathbf{J}_{s}^{p}}{e}$

In arriving at this result we have used the fact that the electron and ion production and loss terms are necessarily the same and we have added the precipitating electrons to the overall electron population. The next step is to use the perpendicular ion and parallel electron momentum Eqs. to arrive at results that can be expressed in terms of conductivities. In the context of our model it is easy to show that the resulting Eq. is

$\frac{\partial\left(\sigma_{p} \delta E_{x}\right)}{\partial x}+\frac{\partial\left(\sigma_{\|} \delta E_{z}\right)}{\partial z}=-\frac{\partial J_{s}^{p}}{\partial z}-E_{x}^{0} \frac{\partial \sigma_{p}}{\partial x}-E_{y}^{0} \frac{\partial \sigma_{h}}{\partial x}$

where we have used $\delta \mathbf{E}$ to describe the $-\nabla \phi$ term that we get from our numerical model. Notice how the electron term containing $E_{y}^{0}$ in Eq. 37 is absorbed in the last term on the right-hand side of Eq. 38. This shows that the Eq. $\nabla \cdot \mathbf{J}=0$ that we use in the text is indeed describing continuity in a frame of reference moving in the $x$ direction at the $E_{y}^{0} / B$ speed. It also stresses that in the presence of such a motion, we need to include a term containing the derivative of the Hall conductivity as a source term. In the present work we have not included that term, i.e., we have used $E_{y}^{0}=0$. This is not a fundamental limitation as long as $\left|E_{x}^{0}\right| \gg\left|E_{y}^{0}\right|$ and since the conductivity derivatives have comparable magnitudes. Furthermore, given that the $E_{y}^{0}$ term can even have either sign (depending on the sign of the arc motion), we have chosen to study the influence of this extra parameter in future studies only. Recall that the main goal of the present work is to introduce our new modeling tool rather than to explore the large number of possibilities that can later be studied with it.

Finally, we note that the effect of perpendicular pressure and temperature gradients is not being considered in the rest of our transport equations. For important diamagnetic correction terms, the horizontal gradient terms would have to be even steeper than those we are considering here, namely, of the order of the ion gyroradius itself (St.-Maurice et al., 1994). In other words, it is reasonable even in the context of our short-scale electrodynamics to consider that horizontal gradients are important only if and when they create polarization electric fields through the electrodynamics described by our work.

\section{References}

Berthelier, J.-J., C. Machard, J.-C. Cerisier, and A. Berthelier, ULF electromagnetic turbulence in the high-latitude topside ionosphere, J. Geophys. Res., 93, 5701-5712, 1988.

Blelly, P.-L., and R. W. Schunk, A comparative study of the timedependent standard 8-, 13- and 16-moment transport formulations of the polar wind, Ann. Geophys., 11, 443-469, 1993.

Blelly, P.-L., A. Robineau, J. Lilensten, and D. Lummerzheim, 8-moment fluid models of the terrestrial high latitude ionosphere between 100 and $3000 \mathrm{~km}$, in Solar terrestrial energy program (STEP): handbook of ionospheric models, ed R. W. Schunk, 53-72, 1996.

Cerisier, J.-C., C. Machard, and R. Pottelette, MHD turbulence generated by time-varying field-aligned currents, J. Geophys. Res., 92, $11225-11230,1987$.

Collis, P. N., I. Häggström, K. Kaila, and M. T. Rietveld, EISCAT radar observations of enhanced incoherent scatter spectra; their relation to red aurora and field-aligned currents, Geophys. Res. Lett., 18, 1031-1034, 1991.

Diloy, P.-Y., A. Robineau, J. Lilensten, P.-L. Blelly, and J. Fontanari, A numerical model of the ionosphere, including the E-region above EISCAT, Ann. Geophys., 14, 191-200, 1996.

Earle, G. D., M. C. Kelley, and G. Ganguli, Large velocity shears and associated electrostatic waves and turbulence in the auroral F-region, J. Geophys. Res., 94, 15 321-15 333, 1989. 
Forme, F. R. E., A new interpretation on the origin of enhanced ion-acoustic fluctuations in the upper atmosphere, Geophys. Res. Lett., 20, 2347-2350, 1993.

Gavrishchaka, V. V., S. B. Ganguli, and G. I. Ganguli, Origin of low-frequency oscillations in the ionosphere, Phys. Rev. Lett., 80, 728-731, 1998.

Hargreaves, J. K., The solar-terrestrial environment, Cambridge Univ Press, Cambridge, 1992.

Kelley, M. C., The Earth's ionosphere: plasma physics and electrodynamics, Academic Press, New York, 1989.

Kivelson, M. G., and C. T. Russell, Introduction to space physics, Cambridge Univ Press, Cambridge, 1995.

Lilensten, J., W. Kofman, J. Wisenberg, E. S. Oran, and C. R. Devore, Ionization efficiency due to primary and secondary photoelectrons: a numerical model, Ann. Geophys., 7, 83-90, 1989.

Lummerzheim, D., and J. Lilensten, Electron transport and energy degradation in the ionosphere: evaluation of the numerical solution, comparison with laboratory experiments and auroral observations, Ann. Geophys., 12, 1039-1051, 1994.

Maggs, J. E., and T. N. Davis, Measurements of the thickness of auroral structures, Planet. Space Sci., 16, 205-209, 1968.

Marklund, G., I. Sandahl, and H. Opgenoorth, A study of the dynamics of a discrete auroral arc, Planet. Space Sci., 30(2), 179-197, 1982.

Rees, M. H., Physics and chemistry of the upper atmosphere, Cambridge Univ Press, Cambridge, 1989.

Rietveld, M. T., P. N. Collis, and J.-P. St.-Maurice, Naturally enhanced ion acoustic waves in the auroral ionosphere observed with the EISCAT 933-MHz radar, J. Geophys. Res., 96, $19291-$ 19 305, 1991.

Robineau, A., P.-L. Blelly, and J. Fontanari, Time-dependent models of the auroral ionosphere above EISCAT, J. Atmos. Terr. Phys., 58, 257-271, 1996.

Schunk, R. W., Mathematical structure of transport equations for multispecies flows, Rev. Geophys. Space Phys., 15, 429-477, 1977.
Schunk, R. W., and A. F. Nagy, Electron temperatures in the F- region of the ionosphere, Rev. Geophys. Space Phys., 16, 355-399, 1978.

Schunk, R. W., and A. F. Nagy, Ionospheres of the terrestrial planets, Rev. Geophys. Space Phys., 16, 813-852, 1980.

Schunk, R. W., and J. C. G. Walker, Transport processes in the F-region of the ionosphere, J. Geophys. Res., 76, 6159-6171, 1971.

Sedgemore-Schulthess, K. J. F., M. Lockwood, T. S. Trondsen, B. S. Lanchester, M. H. Rees, D. A. Lorentzen, and J. Moen, Coherent ESR spectra from the dayside cusp/cleft and their implications for transient field-aligned currents, J. Geophys. Res., 104, 24 613-24 624, 1999.

St.-Maurice, J.-P., and P. J. Laneville, Reaction rate of $\mathrm{O}^{+}$with $\mathrm{O}_{2}, \mathrm{~N}_{2}$ and $\mathrm{NO}$ under highly disturbed auroral conditions, J. Geophys. Res., 103, 17 519-17 521, 1998.

St.-Maurice, J.-P., and D. G. Torr, The effect of relative speed distributions on the reaction rates of $\mathrm{O}^{+}$with $\mathrm{N}_{2}, \mathrm{O}_{2}$ and $\mathrm{NO}$ in the thermosphere, J. Geophys. Res., 83, 969-977, 1978.

St.-Maurice, J.-P., E. Winkler, and A. M. Hamza, Ionospheric ion velocity distributions and associated transport properties in the presence of auroral electric field gradients, J. Geophys. Res., 99, 19 527-19 549, 1994.

St.-Maurice, J.-P., W. Kofman, and D. James, In situ generation of intense parallel electric fields in the lower ionosphere, J. Geophys. Res., 101, 335-356, 1996.

Trondsen, T. S., High spatial and temporal resolution: auroral imaging, Ph.D. thesis, Univ Tromso, Tromso, Norway, 1998.

Trondsen, T. S., and L. L. Cogger, High-resolution television observations of black aurora, J. Geophys. Res., 102, 363-378, 1997.

Whalund, J.-E., F. R. E. Forme, H. J. Opgenoorth, and M. A. L. Persson, Scattering of electromagnetic waves from a plasma: enhanced ion-acoustic fluctuations due to ion-ion two-stream instabilities, Geophys. Res. Lett., 19, 1919-1922, 1992. 